\title{
First measurements of a magnetically driven fast-ion loss detector on ASDEX Upgrade
} \author{
J. Galdon-Quiroga ${ }^{d}$, A. Kovacsik ${ }^{\mathrm{e}}$, P. Leitenstern ${ }^{\mathrm{d}}$, T. Lunt ${ }^{\mathrm{d}}$, \\ MST12 Teams \\ ${ }^{a}$ Department of Mechanical Engineering and Manufacturing, \\ University of Seville Spain \\ ${ }^{b}$ Centro Nacional de Aceleradores (CNA), \\ Universidad de Sevilla, CSIC, Junta de Andalucía \\ ${ }^{c}$ Department of Atomic, Molecular and Nuclear Physics, \\ University of Seville, Spain \\ ${ }^{d}$ Max-Planck-Institut für Plasmaphysik, \\ Garching, Germany \\ ${ }^{e}$ Budapest University of Technology and Economics, \\ Budapest, Hungary \\ ${ }^{f}$ Wigner RCP, \\ Budapest, Hungary \\ E-mail: jgonzalez62@us.es
}

J. Gonzalez-Martinn ${ }^{\mathrm{a}, \mathrm{b}, *}$, M. Garcia-Munoz ${ }^{\mathrm{b}, \mathrm{c}}$, A. Herrmann ${ }^{\mathrm{d}}$, J. Ayllon-Guerola ${ }^{\mathrm{a}, \mathrm{b}}$, J.F. Rivero-Rodriguez ${ }^{\mathrm{a}, \mathrm{b}}$, B. Sieglin ${ }^{\mathrm{d}}$, S. Zoletnik ${ }^{\mathrm{f}}$, J. Dominguez ${ }^{\mathrm{a}}$, AUG $^{1}$ and

ABSTRACT: A new scintillator-based fast-ion loss detector (FILD) has been deployed $\sim 45^{\circ}$ below the midplane of the ASDEX Upgrade tokamak. Port unavailability at this remote location requires an in-situ magnetically driven manipulator to move the diagnostic head horizontally through the scrape-off layer (SOL). The linear displacement is produced by an externally energized coil, whose magnetic dipole tries to align with the toroidal component of the tokamak magnetic field. The insertion is given by force balance between a retaining spring and the energized solenoid, whose current is regulated in real-time, opening the possibility of self-adaptive real-time control of the probe head location based on its temperature. The diagnostic head contains a scintillator screen, Faraday cup, thermocouple and collimator systems. The scintillator image is transferred to a vacuum window using a 3.5 meters quartz image guide. The light acquisition system is composed by a charge coupled device (CCD) camera, for high velocity-space resolution, and by an $8 \times 4$ channels avalanche photo diode (APD) camera, for high temporal resolution (up to $2 \mathrm{MHz}$ ). First measurements of magnetohydrodynamic (MHD) induced fast-ion losses and radially resolved fast-ion losses are presented.

KEYWORDS: detector, scintillator, FILD, tokamak, fast-ion, losses, MHD, magnetic drive, real time.

\footnotetext{
${ }^{1}$ See authors list in A Kallenbach et al., Nucl. Fusion 53, 102015 (2017).

${ }^{2}$ See authors list in H. Meyer et al., Nucl. Fusion 57, 102014 (2017).
} 


\section{Contents}

1. Introduction 1

2. Diagnostic Setup 2

2.1 Probe head design and location 2

2.2 Optics relay and light acquisition systems 2

3. Magnetically Driven Manipulator 3

4. First measurements 4

4.1 NBI source scan 4

4.2 NTM induced fast-ion losses 4

4.3 Radial scan of fast-ion losses 5

$\begin{array}{ll}\text { 5. Conclusions and future applications } & 6\end{array}$

\section{Introduction}

Good fast-ion confinement (including fusion-born alpha particles) is of paramount importance to obtain an ignited plasma in ITER and ensure device integrity. In present devices, suprathermal ions generated by auxiliary heating systems such as Neutral Beam Injector (NBI) and Ion Cyclotron Resonance Frequency (ICRF) heating are used to study fast-ion radial
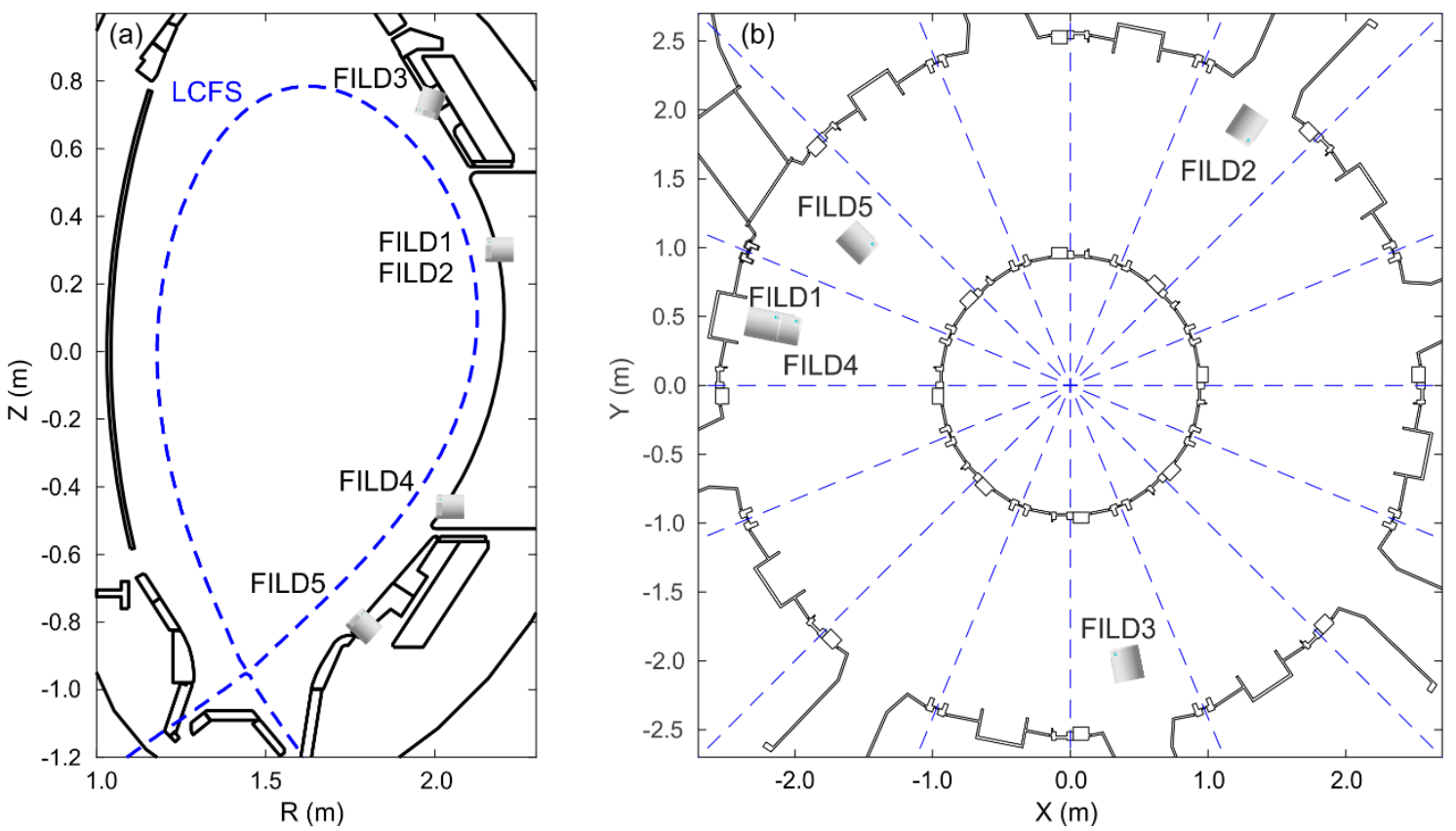

Figure 1. Poloidal (a) and toroidal (b) distribution of the five FILD systems installed on the ASDEX Upgrade tokamak, including the recently installed magnetically driven FILD4. 
transport produced by toroidal magnetic field ripple, externally applied 3D fields [1], and different branches of magnetohydrodynamic (MHD) instabilities [2]. To help validating the theoretical models that describe fast-ion transport, scintillator-based fast-ion loss detectors (FILDs) [3] provide velocity-space measurements with Alfvénic temporal resolution. FILD covers the entire velocity-space, however, since its measurements are localized at a certain position, wall distribution is missing and multiple FILDs are required to fully characterize fastion loss distribution. This motivated the installation of a suite of 4 FILDs in the ASDEX Upgrade tokamak (AUG), distributed at different toroidal locations and covering the entire low field side as shown in figure 1 [4]. Aiming at improving the poloidal coverage of this suite, a fifth FILD (labelled FILD4) was deployed in a remote position $\left(\sim 45^{\circ}\right.$ below the mid-plane) where port unavailability prevents from using a conventional FILD design that is driven by an out-vessel step motor. An in-situ concept $[5,6,7,8]$ in which probe head motion is provided by an externally energized coil, is applied, allowing easier vessel installation, radial fast-ion loss profiles and opening the door for real-time control of probe head insertion, to use FILD in a wider range of operation parameters.

This paper is structured as follows: In section 2, the diagnostic setup is described along its probe head, relay lenses and light acquisition systems. Section 3 is devoted to the description of its dedicated manipulator, including electromotive damping and the algorithm to measure probe head insertion. Section 4 discusses initial measurements, including MHD induced fast-ion losses and radial scan of fast-ion losses in the SOL. Conclusions and future work to exploit this diagnostic are discussed in section 5.

\section{Diagnostic set-up}

The working principle of this new probe is based on the first FILD installed in AUG back in 2005 [3]: incoming fast-ions are collimated by the relative arrangement of the D-shaped graphite cup, collimator slit and scintillator coated plate, which emits light when ions impinge on it. Fast-ion gyro-orbits determine the strike point on the scintillator plate, enabling us to

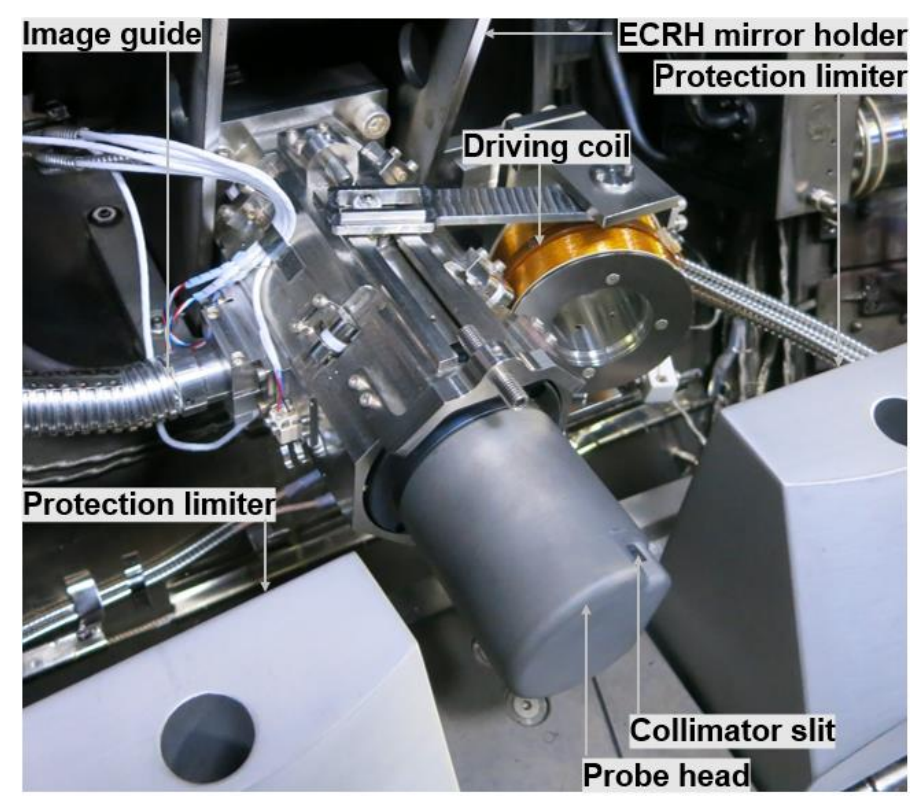

Figure 2. The new FILD installed on AUG. The system is clamped to a mirror holder from an ECRH launcher. Probe head, collimator slit, driving coil, image guide, and protection limiters can be identified. 
identify fast-ion gyroradii (and thus energy) and pitch angle. The short decay time (490 ns) of the scintillating phosphate used in the AUG FILD system (commercially known as TG-Green) makes it possible to identify fast-ion losses induced by high-frequency MHD instabilities.

\subsection{Probe head design and location}

The FILD D-shape graphite cup (diameter $\sim 7 \mathrm{~cm}$ ) is tungsten-coated like all the other plasma facing components at AUG. It contains the most sensitive elements, such as collimator, scintillator plate, calibration lamp and thermocouple. The calibration lamp helps to align the out-vessel camera chip with respect to scintillator plate edges. The thermocouple measures the inner temperature of the probe head while a camera from the AUG video real time (VRT) system tracks the temperature of the outer surface. Current generated in the scintillator plate by striking ions is extracted and can be interpreted as an absolute ion flux by subtracting the background signal from an insulated plate behind the scintillator.

The entire FILD system is placed $\sim 45^{\circ}$ below the AUG mid-plane, about 2 meters away from the $93 \mathrm{keV}$ NBI box and below the midplane manipulator (in which FILD1 is installed). The entire system is clamped to an ECRH launcher mirror holder as shown in figure 2. FILD has been designed such that it never interferes with the ECRH line of sight. An external steel shield protects the outer sensitive components (such as the driving coil) from plasma radiation.

\subsection{Optics relay and light acquisition systems}

The light pattern produced by collimated fast-ions is focused by a lens located at $15 \mathrm{~mm}$ from the scintillator plate as depicted in figure 3 . The diameter of this lens is $5 \mathrm{~cm}$ and is drilled on its edge to fit the calibration lamp and the thermocouple. A set of 2 lenses and a mirror deviates light 90 degrees towards an image guide. The moving rod has a $26 \mathrm{~mm}$ slot used to accommodate a fixed structure holding the mirror. The image guide contains $40 \times 50$ quartzmade fibers and transports the light 3.5 meters from FILD towards an anti-reflection coated vacuum window located in an upper port. The light beam passing through the vacuum window is split by a drilled mirror into two light acquisition systems. The light passing through the drill reaches a charge couple device (CCD) camera [9] acquiring at 50 frames/s (and exposure time of 20 milliseconds) with a resolution of $480 \times 640$ pixels. In addition, the light reflected by the mirror, is recorded by a $4 \times 8$ channels avalanche photo diode (APD) camera [10] at high temporal resolution (up to $2 \mathrm{MHz}$ ). This drilled mirror solution was found to be more effective than a beam splitter. Due to the strong demagnification to the CCD camera sensor, the Numerical Aperture for the CCD camera is limited. With this solution all the light which cannot be made use of by the CCD camera is directed to the APD camera.

Both cameras are connected to their respective data converter using two 5 meters ethernet cables, which are insulated against ICRF cross talk by a copper mesh grounded to a vessel

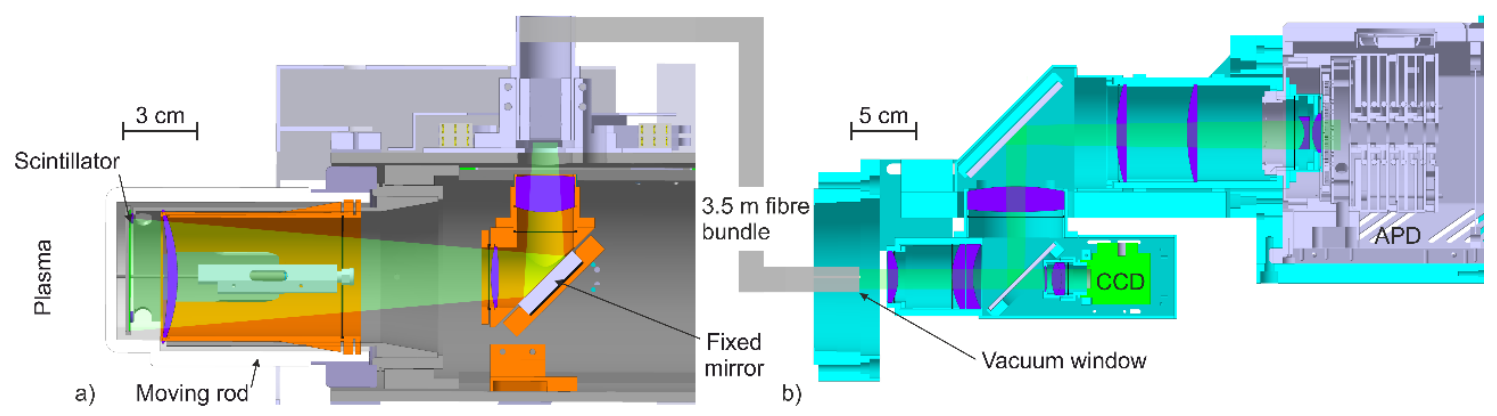

Figure 3. In-vessel (a) and out-vessel (b) optics of FILD. Light path is depicted in green while lenses are in purple. In the out-vessel optics relay, CCD and APD cameras are visible. 
potential on one end. Each data converter sends the signal to the FILD Linux servers located in the control room using a $1 \mathrm{Gbit}$ optic connection, which is unaffected by ICRF noise.

\section{Magnetically driven manipulator}

Magnetically driven manipulators are often used for reciprocating Langmuir probes $[6,7,8]$. The same working principle is applied to the FILD manipulator, whose maximum stroke is $80 \mathrm{~mm}$, having the collimator slit exposed to fast-ion orbits when inserted $\sim 40 \mathrm{~mm}$ from its parking position. A retaining spring holds the system back when not used. Coil rotation is given by balance between retaining spring torque, coil torque and coil and probe head inertias [5]. The coil (placed on an electrically insulated holder) is energized by a power supply controlled in real-time by the discharge control system. The system is potentially capable of reacting to events that could harm the probe head (such as overheating and plasma displacements).
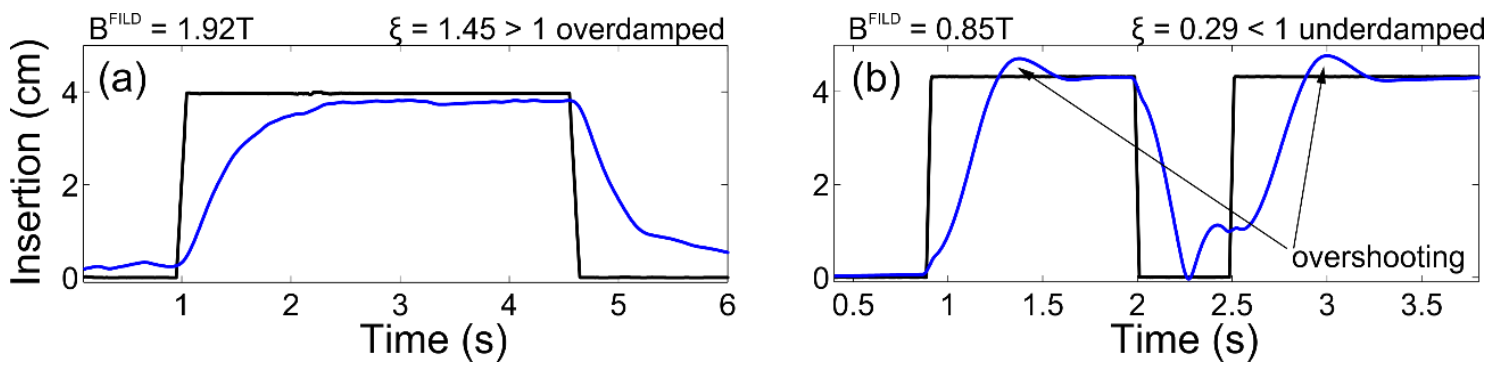

Figure 4. Head target (black) and measured (blue) insertion for the overdamped (a) and underdamped regimes (b). In the overdamped regime, the probe head takes longer to reach the target position. In the underdamped regime, the probe head overshoots, which could result in unexpected heat loads on the head.

Coil rotation is inferred from coil inductance using a similar algorithm described in Ref. 5. Furthermore, from linearized motion equations one can calculate the damping ratio, $\xi$, (following an approach similar to Ref 6. equation 12) and observe that it depends quadratically on the magnetic field at the coil location:

$$
\left.\xi\right|_{\theta_{0}=0}=\frac{(N A B)^{2}}{2 R \sqrt{1^{2} K\left(\iota+l^{2} m\right)}}
$$

where $\mathrm{N}(250)$ is the number of coil windings, $A\left(0.054 \mathrm{~m}^{2}\right)$ is the area enclosed by each winding, B is the magnetic field at the FILD position, $\mathrm{R}(13 \Omega)$ is the resistance of the coil, 1 $(10.5 \mathrm{~cm})$ is the distance between the coil rotation axis and the probe head axis, $\mathrm{K}$ is the angular resistance of the linear spring, $\mathrm{l}$ is the coil inertia and $\mathrm{m}$ is the mass of the probe head and sliding rod. Figure 4 depicts the measured probe head movement under two different magnetic fields. In the first case, a larger magnetic field at FILD position $(B=1.92 \mathrm{~T})$ produces larger damping ratio $(\xi=1.45)$, therefore the system is overdamped, taking longer to reach the target position. In plot $(\mathrm{b})$, lower magnetic field $(\mathrm{B}=0.85 \mathrm{~T})$ produces faster movement, however, the system is underdamped $(\xi=0.85)$, producing overshooting which jeopardizes the integrity of the probe head. The best scenario for operating this manipulator is determined to be about $\mathrm{B}_{\mathrm{t}} \sim$ $1.8 \mathrm{~T}$, which will produce critical damping (i.e. improved control of probe head position).

\section{First measurements}

In the last opening of the AUG vessel in 2018, FILD4 was installed, its magnetically driven manipulator was commissioned during shots in which there was no plasma (that could 
harm the graphite cup), but the toroidal magnetic field needed to drive the probe was available. Once FILD head was demonstrated to move as desired, dedicated experiments were performed to test the whole diagnostic. These discharges had a plasma current of $\mathrm{I}_{\mathrm{p}}=0.6 \mathrm{MA}$, a toroidal magnetic field of $\mathrm{B}_{\mathrm{t}}=2.4 \mathrm{~T}$ and were performed right after a boronization, aiming at low densities $\left(\mathrm{n}_{\mathrm{e} 0} \sim 3.5 \times 10^{19}\right.$ particles $\left./ \mathrm{m}^{3}\right)$ to reduce collisionallity which will ultimately increase the fast-ion content. Only one NBI source was turned on at each time, allowing to disentangle the fast-ion loss signal produced by each beam. In addition (to compensate the low, $2.5 \mathrm{MW}$, of NBI heating) 5 gyrotrons were turned on, generating $2.6 \mathrm{MW}$ of ECRH which avoided reaching the H-L transition at the end of each NBI phase. The position of the last closed flux surface was feedback controlled by the discharge control system keeping the plasma edge more than $5 \mathrm{~cm}$ away from the FILD probe on its innermost position.

\subsection{NBI source scan}

The FILD collimator slit was exposed about $5 \mathrm{~mm}$ beyond the limiters, allowing fast-ion orbits to enter the probe head. Consecutive NBI phases of $200 \mathrm{~ms}$ were programmed on each source. $100 \mathrm{~ms}$ (about one fast-ion slowing down time) were left between each NBI phase to observe a drop in the fast-ion content. Figure 5(a) shows how both the CCD and APD cameras record an increase in the signal level each time an NBI source starts producing fast-ions. Beams from the $60 \mathrm{keV}$ injector (sources labelled as \#2, \#3, and \#4 in figure 5) were shut down after 75 ms. These short phases, along with the fact that those injectors are located far from FILD, explain the low value of the measured signal.
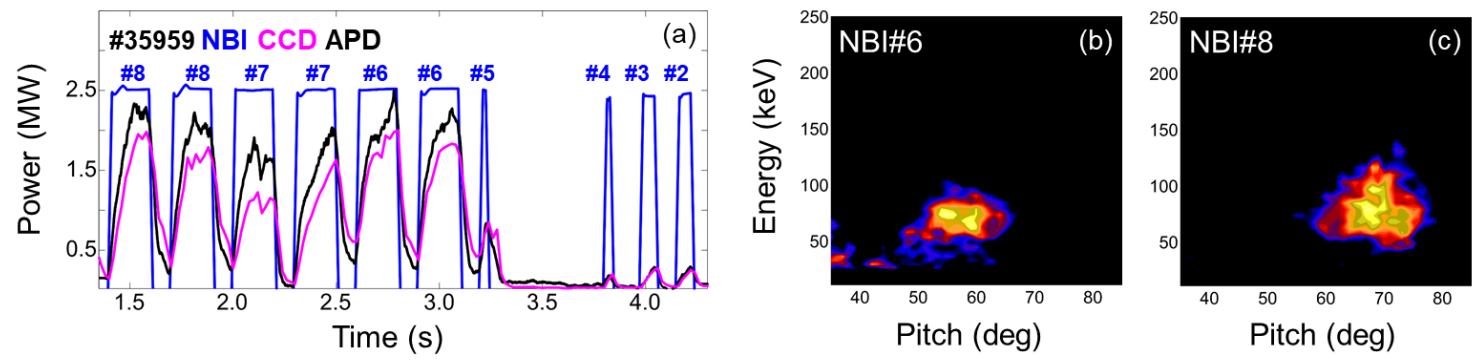

Figure 5. (a) Fast-ion loss signal generated by different NBI sources and recorded by both CCD and APD cameras. Fast-ion loss velocity space pattern produced by tangential beam \#6 (b) and radial beam \#8 (c).

From the scintillator plate images obtained by the CCD camera, pitch angle, $\operatorname{arcos}\left(\mathrm{v}_{\|} / \mathrm{v}\right)$, and gyroradii measurements can be reconstructed by means of triangulation techniques over a strike map calculated by the FILDSIM code as described in Ref. [11]. Fast-ion reaching the

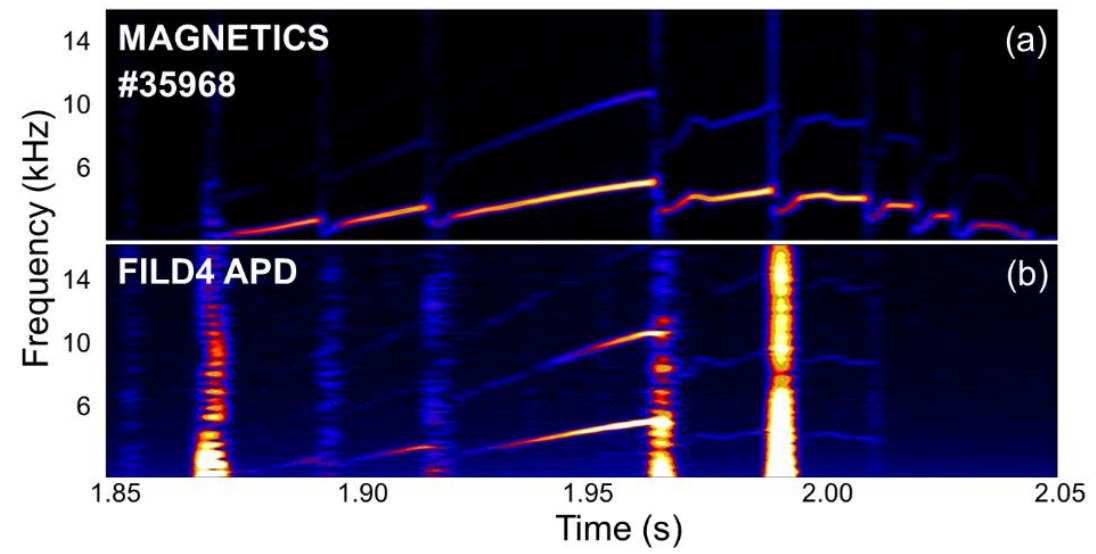

Figure 6. An NTM observed in (a) Mirnov coil spectrogram (b) FILD APD channel 19 spectrogram. 
FILD probe are assumed to be deuterium (which are injected by the NBI system) allowing to infer their energy, $\sim 93 \mathrm{keV}$, matching the NBI main injection energy. In figures 5(b) and 5(c) one can observe that the pitch angle of ions deposited on FILD depends on the applied beam. For radial beams (\#5 and \#8) trapped orbits (pitch $70^{\circ}$ ) are measured while for tangential beams (\#6 and \#7) the particles reaching FILD are passing (pitch $\sim 55^{\circ}$ ).

\subsection{MHD induced fast-ion losses}

A neoclassical tearing mode (NTM) was created, rotating as a rigid body at the same frequency as the background plasma, as shown in the Mirnov coil signal spectrogram of figure 6(a). The analysis of the signals from different Mirnov coils allows identifying the toroidal $(n=$ 1) and poloidal $(m=3)$ mode numbers of this instability. Fast soft $x$-ray measurements determine that the instability is located around a normalized radius of $\rho_{\mathrm{pol}}=0.6$. Figure $6(\mathrm{~b})$ shows the spectrogram from one of the APD channels that measures the beam-modulated losses. Fast-ion losses produced by the NTM are observed. FILD slit remains fixed about R $1.98 \mathrm{~m}$ during that time window. After the beam is turned off at 2.0 seconds, the NTM is still visible on the Mirnov coil spectrogram but disappear from the FILD spectrogram. This is explained by the fact that the beam stops injecting prompt losses that interact with the NTM and reach the FILD probe.

\subsection{Radial scan of fast-ion losses}

FILD4 was programmed to swing back and forth during the different NBI phases. Position measurements based on coil induction show that the probe head is driven as desired and retracts to its parking position by the end of the plasma pulse. The covered radial distance is about 40 $\mathrm{mm}$ with an average velocity $\left\langle\mathrm{V}_{\mathrm{FILD}}\right\rangle \sim 0.1 \mathrm{~mm} / \mathrm{ms}$. The APD camera signal is plotted against collimator slit position, obtaining radial profiles of fast-ion losses in the scrape-off layer. In figure 7(a) fast-ion loss profiles generated by beam \#7 are shown. The signal is observed to increase linearly after the collimator slit is inserted beyond the protection limiters. The large spikes in the traces correspond to losses induced by edge-localized modes (ELMs) [12].
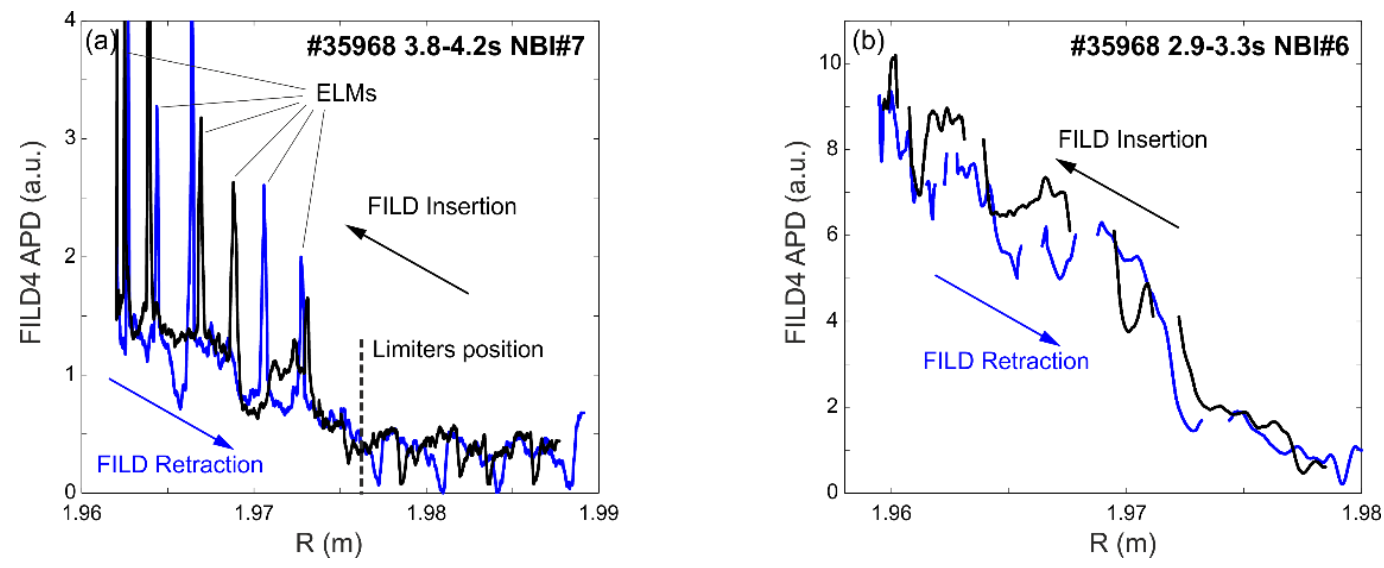

Figure 7. Fast-Ion radial scan obtained by swinging the probe during two different NBI phases. ELMs and limiter shadow are shown in (a) while are removed in (b) to better observe the linear part or the curve.

In figure 7(b), when beam \#7 was replaced by beam \#6, APD signal during ELMs and shadow region is removed, so the linear dependence of fast-ion losses on the radial position can be easily observed. For both neutral beam injectors, the APD signal obtained during the FILD insertion matches the signal when the FILD probe is being retracted. 


\section{Conclusions and future applications}

A new magnetically driven scintillator-based fast-ion loss detector has been commissioned on ASDEX Upgrade. This in-situ system enables fast-ion loss measurements in a poloidal location impossible to cover using conventional manipulators. Prompt and MHD induced fastion losses are identified. The FILD probe was moved during one plasma shot, showing the capability of obtaining radially resolved fast-ion loss profiles. The system has an average speed of $\left\langle\mathrm{V}_{\mathrm{FILD}}\right\rangle \sim 0.1 \mathrm{~mm} / \mathrm{ms}$. The velocity could be eventually increased to $\left\langle\mathrm{V}_{\mathrm{FILD}}\right\rangle \sim 1 \mathrm{~mm} / \mathrm{ms}$ by simply tuning the applied voltage curve. This system opens the possibility to employ a real-time FILD insertion control based on probe head temperature, which will considerably expand its operational window. Furthermore, radially resolved light ion beam probe technique [13] is expected to be capable of radially probe the fast-ion orbit deflection produced by different kinds of plasma perturbations (NTMs, TAEs, Edge Resonant Transport Layer [14]).

\section{Acknowledgments}

The authors would like to show their gratitude to Wolfgang Popken and the entire AUG Vessel Team for their contribution to this work.

This work has been carried out within the framework of the EUROfusion Consortium and has received funding from the Euratom research and training programme 2014-2018 and 20192020 under Grant Agreement No. 633053. The views and opinions expressed herein do not necessarily reflect those of the European Commission. This research also received funding from the Spanish Ministry of Science under grant No FPU15/06074.

\section{References}

[1] M. Garcia-Munoz et al., Plasma Phys. Control. Fusion, 55, (2013).

[2] S. J. Zweben et al., Nucl. Fusion 30, 1551 (1990).

[3] M. Garcia-Munoz, et al., Rev. Sci. Instrum. 80, 53503 (2009).

[4] J. Gonzalez-Martin et al., Rev. Sci. Instrum. 89, 101106 (2018).

[5] J. Ayllon et al., Rev. Sci. Instrum. 87, 11E705 (2016).

[6] J.P. Gunn et al., Rev. Sci. Instrum. 82, 123505 (2011).

[7] A. Schmid et al., Rev. Sci. Instrum. 78, 053502 (2007).

[8] C. K. Tsui et al., Rev. Sci. Instrum. 83, 10D723 (2012).

[9] G. Náfrádi, et al., Nucl. Instrum. and Method in Phys. Res. 21, 770 (2015).

[10] E. Dunai et al., Rev. Sci. Instrum. 81, 103503 (2010).

[11] J. Galdon-Quiroga et al, Plasma Phys. Control. Fusion 60, 105005 (2018).

[12] J. Galdon-Quiroga et al, Nucl. Fusion 59, 066016 (2019).

[13] X. Chen et al., Rev. Sci. Instrum. 85, 11E701 (2014).

[14] L. Sanchis et al., Plasma Phys. Control. Fusion 61, 014038 (2019). 